\title{
ON ITERATIVE REGULARIZATION METHODS FOR VARIATIONAL INEQUALITIES OF THE SECOND KIND WITH PSEUDOMONOTONE OPERATORS ${ }^{1}$
}

\author{
I. B. BADRIEV, O. A. ZADVORNOV, AND L. N. ISMAGILOV \\ Department of Computational Mathematics and Cybernetics, Kazan State University \\ Kremlevskaya Str., 18, 420008, Kazan, Russia
}

\begin{abstract}
Variational inequalitiy of the second kind
$\left\langle A_{0} u, \eta-u\right\rangle+F_{1}(\eta)-F_{1}(u) \geqslant\langle f, \eta-u\rangle$

with a pseudomonotone operator $A_{0}$ and a convex non-differentiable functional $F_{1}$ in the Banach or Hilbert space is considered. A "semi-implicit" iterative method with explicit approximation of the operator $A_{0}$ and regularized functional $F_{\varepsilon}$ for its solution is studied.
\end{abstract}

2000 Mathematics Subject Classification: 65N22; 65N10; 65N06; 65N55.

Keywords: iterative method, variational inequalitiy of the second kind, pseudomonotone operator, convex non-differentiable functional, iterative regularization.

\section{Introduction}

In this paper, we study variational inequalities of the second kind with a pseudomonotone operator and convex non-differentiable functional in Banach spaces that occur, in particular, in describing stationary seepage processes and problems of defining soft shell equilibrium. To solve a variational inequality, a two-level method of iterative regularization is suggested. It allows to reduce the solution of the original variational inequality and the regularized functional to the solution of the variational inequality with a duality operator. The duality operator has better properties compared to the original operator. Iterative processes of this type have already been considered earlier (see, e.g., $[1,2,4,5,8,11,14,16]$ ). However, these investigations considered only cases with strongly monotone operators or maximal monotone operators.

We investigate the convergence of an iterative process. It is proved that the iterative sequence is bounded and any of its weak limit points are a solutions of the original inequality. In the case of a Hilbert space under an additional limitation, a weak convergence of the whole iterative sequence is proved.

Note that in [6] we investigated an iterative method for solving the same variational inequalities without regularization. At each step of this method it is necessary to solve a

\footnotetext{
${ }^{1}$ The work was supported by the Russian Basic Research Foundation, grants 01-01-00616, 03-01-00380 and the Ministry of Education of the Russian Federation, grant E02.-1.0-189.
} 
convex non-differentiable problem, which causes an additional difficulty in implementation of the algorithm. The method suggested in this paper leads to a much easier problem (in the sense of its implementation) if the regularized functional becomes differentiable.

The general results obtained are applied to a stationary seepage problem for an incompressible liquid that follows a discontinuous seepage law with the limit gradient (see $[3,12,16])$. Some results regarding properties of the solution of seepage problems are also obtained.

The proposed algorithm can be applied to the solution of a wide class of problems with non-monotone operators. One of the most important examples is the problem of the soft shells theory (see, e.g., [15]) where operator is the sum of the nonlinear monotone operator and the linear self-adjoint indefinite one.

\section{The case of the Banach space}

Let $V$ be a reflexive Banach space with a uniformly convex conjugate space $V^{*},\langle\cdot, \cdot\rangle$ is the duality relation between $V$ and $V^{*}, M$ is the convex closed set in $V, A_{0}: V \rightarrow V^{*}$ is the pseudomonotone, coercive operator that satisfies the conditions (see $[3,9]$ )

$$
\begin{aligned}
& \int_{0}^{1}\left(\left\langle A_{0}(t(u+v)), u+v\right\rangle-\left\langle A_{0}(t u), u\right\rangle\right) d t=\int_{0}^{1}\left\langle A_{0}(u+t v), v\right\rangle d t \forall u, v \in V, \\
&\left\|A_{0} u-A_{0} v\right\|_{V^{*}} \leqslant \mu(R) \Phi\left(\|u-v\|_{V}\right) \quad \forall u, v \in V,
\end{aligned}
$$

where $R=\max \left\{\|u\|_{V},\|v\|_{V}\right\}, \mu$ is the nondecreasing function on $[0,+\infty), \Phi$ is the strictly increasing continuous function on $[0,+\infty)$ such that $\Phi(0)=0, \Phi(\xi) \rightarrow+\infty$ as $\xi \rightarrow+\infty$.

It will be recalled (see [13]) that the operator $A_{0}$ is pseudomonotone if $A_{0}$ is a bounded operator and from the weak convergence of the sequence $\left\{u_{n}\right\}_{n=1}^{\infty} \in V$ to $u$ in $V$ and inequality

$$
\limsup _{n \rightarrow \infty}\left\langle A_{0} u_{n}, u_{n}-u\right\rangle \leqslant 0
$$

it follows that

$$
\liminf _{n \rightarrow \infty}\left\langle A_{0} u_{n}, u_{n}-v\right\rangle \geqslant\left\langle A_{0} u, u-v\right\rangle \quad \forall v \in V .
$$

By the operator coerciveness is meant the realization of the following inequality:

$$
\left\langle A_{0} u, u\right\rangle \geqslant \rho\left(\|u\|_{V}\right)\|u\|_{V} ; \quad \lim _{\xi \rightarrow+\infty} \rho(\xi)=+\infty .
$$

Let later $F_{1}: V \rightarrow R^{1}$ be a convex (in general, non-differentiable) weak lower semicontinuous functional.

We define the functional $F: V \rightarrow R^{1}$ by the following relation:

$$
F(u)=F_{0}(u)+F_{1}(u)-\langle f, u\rangle, \quad F_{0}(u)=\int_{0}^{1}\left\langle A_{0}(t u), u\right\rangle d t, \quad f \in V^{*} .
$$

Here from (1), (6) it follows that for any $u, v \in V$ the following equality is valid:

$$
F(u)-F(v)=\int_{0}^{1}\left\langle A_{0}(v+t(u-v)), u-v\right\rangle d t+F_{1}(u)-F_{1}(v)-\langle f, u-v\rangle .
$$


We observe that by virtue of the convexity of the functional $F_{1}$ and condition (5) the functional $F$ is coercive (see, e.g., [7]).

Now we consider the problem of searching the element $u \in M$, which is a solution of the following variational inequality of the second kind:

$$
\left\langle A_{0} u, \eta-u\right\rangle+F_{1}(\eta)-F_{1}(u) \geqslant\langle f, \eta-u\rangle \quad \forall \eta \in M .
$$

We consider for $\varepsilon>0$ a convex functional $F_{\varepsilon}$ satisfying the conditions

$$
\begin{array}{lrr}
\left|F_{\varepsilon}(\eta)-F_{1}(\eta)\right| \leqslant c(\varepsilon), & \forall \eta \in V, & \lim _{\varepsilon \rightarrow 0} c(\varepsilon)=0 . \\
\left|F_{\varepsilon}(v)-F_{\varepsilon}(u)\right| \leqslant \gamma^{*}\|v-u\|_{V} & \forall u, v \in V, & \gamma^{*}>0 .
\end{array}
$$

From (9), (10) it follows that the functional $F_{1}$ is Lipshitz continuous with the constant $\gamma^{*}$.

In order to solve problem (8), we consider the following iterative process which permits reducing it to a variational inequality of the second kind with a duality operator and a regularized functional whose solution can be obtained by the known methods (see, e.g., $[8,10,14,19])$. It should be noted that the approach under consideration is very useful when the regularized functional $F_{\varepsilon}$ becomes differentiable.

Let $u_{0}$ be an arbitrary element of $M$. For $n=0,1,2, \ldots$ we define $u_{n+1} \in M$ as a solution of the variational inequality

$$
\left\langle J\left(u_{n+1}-u_{n}\right), v-u_{n+1}\right\rangle+\tau\left(F_{\varepsilon_{n}}(v)-F_{\varepsilon_{n}}\left(u_{n+1}\right)\right) \geqslant \tau\left\langle f-A_{0} u_{n}, v-u_{n+1}\right\rangle \quad \forall v \in M,
$$

$J: V \rightarrow V^{*}$ is the duality operator generated by the function $\Phi$ which satisfies the conditions $($ see $[13])$

$$
\langle J v, v\rangle=\|J v\|_{V^{*}}\|v\|_{V}=\Phi\left(\|v\|_{V}\right)\|v\|_{V} \quad \forall v \in V,
$$

the sequence $\left\{\varepsilon_{n}\right\}_{n=1}^{\infty}$ satisfies the condition

$$
\sum_{n=1}^{+\infty} c\left(\varepsilon_{n}\right)=\sigma<+\infty,
$$

$\tau>0$ is the iterative parameter that satisfies the condition

$$
0<\tau<\min \left\{1,1 / \mu_{0}\right\}, \quad \mu_{0}=\mu\left(R_{0}+\Phi^{-1}\left(R_{1}+\gamma^{*}\right)\right),
$$

where

$$
R_{0}=\sup _{u \in S_{0}}\|u\|_{V}, \quad R_{1}=\sup _{u \in S_{0}}\left\|A_{0} u-f\right\|_{V^{*}}, \quad S_{0}=\left\{u \in M: F(u) \leqslant F\left(u_{0}\right)+2 \sigma\right\} .
$$

We observe that from the coerciveness of the functional $F$ it follows that $R_{0}<+\infty$, while from the boundedness of the operator $A_{0}$ we have that $R_{1}<+\infty$. Thus, $\mu_{0}<+\infty$, i.e., the iterative process (11) is well defined.

The solvability of the variational inequalities (8), (11) follows from the results of $[13$, p. 265], [7, p. 52].

The following result is valid.

Theorem 2.1. Let condition (13) be satisfied. Then the iterative sequence $\left\{u_{n}\right\}_{n=1}^{\infty}$ constructed according to (11) is bounded in $V$ and all of its weakly limited points are solutions of problem (8). 
Proof. Let us first prove the boundedness of the iterative sequence and check that the following condition is satisfied:

$$
\left\{u_{n}\right\}_{n=1}^{\infty} \subset S_{0},
$$

and hence $\left\|u_{n}\right\| \leqslant R_{0}, \quad n=0,1,2, \ldots$

By definition, $u_{0} \in S_{0}$. Let $u_{n} \in S_{0}$, then we prove that $u_{n+1} \in S_{0}$. By setting in (11) $v=u_{n}$ and using (10), (12) and $\tau \leqslant 1$, we obtain that

$$
\begin{aligned}
\Phi\left(\left\|u_{n}-u_{n+1}\right\|_{V}\right)\left\|u_{n}-u_{n+1}\right\|_{V} & =\left\langle J\left(u_{n+1}-u_{n}\right), u_{n+1}-u_{n}\right\rangle \\
& \leqslant \tau\left[\left\langle f-A_{0} u_{n}, u_{n+1}-u_{n}\right\rangle+F_{\varepsilon_{n}}\left(u_{n}\right)-F_{\varepsilon_{n}}\left(u_{n+1}\right)\right] \\
& \leqslant\left[\left\|A_{0} u_{n}-f\right\|_{V^{*}}+\gamma^{*}\right]\left\|u_{n}-u_{n+1}\right\|_{V},
\end{aligned}
$$

and using a strict increase of $\Phi$ we have

$$
\left\|u_{n}-u_{n+1}\right\|_{V} \leqslant \Phi^{-1}\left(\left\|A_{0} u_{n}-f\right\|_{V^{*}}+\gamma^{*}\right)
$$

Furthermore, according to (2), for $t \in[0,1]$ we have

$$
\begin{aligned}
\mid\left\langle A_{0}\left(u_{n+1}+t\left(u_{n}-u_{n+1}\right)\right)\right. & \left.-A_{0} u_{n},\left(u_{n}-u_{n+1}\right)\right\rangle \mid \\
& \leqslant \mu\left(R_{*}\right) \Phi\left(\left\|(t-1)\left(u_{n}-u_{n+1}\right)\right\|_{V}\right)\left\|u_{n}-u_{n+1}\right\|_{V} \\
& \leqslant \mu\left(R_{*}\right) \Phi\left(\left\|u_{n}-u_{n+1}\right\|_{V}\right)\left\|u_{n}-u_{n+1}\right\|_{V}
\end{aligned}
$$

where $R_{*}=\max \left\{\left\|u_{n+1}+t\left(u_{n}-u_{n+1}\right)\right\|_{V},\left\|u_{n}\right\|_{V}\right\}$.

On the other hand, by virtue of (16)

$$
\begin{aligned}
\left\|u_{n+1}+t\left(u_{n}-u_{n+1}\right)\right\|_{V}-\left\|u_{n}\right\|_{V} & \leqslant\left\|(1-t)\left(u_{n}-u_{n+1}\right)\right\|_{V} \leqslant\left\|u_{n}-u_{n+1}\right\|_{V} \\
& \leqslant \Phi^{-1}\left(\left\|A_{0} u_{n}-f\right\|_{V^{*}}+\gamma^{*}\right) .
\end{aligned}
$$

Therefore

$$
R_{*} \leqslant\left\|u_{n}\right\|_{V}+\Phi^{-1}\left(\left\|A_{0} u_{n}-f\right\|_{V^{*}}+\gamma^{*}\right) \leqslant R_{0}+\Phi^{-1}\left(R_{1}+\gamma^{*}\right) .
$$

Since $\mu$ is a nondecreasing function, $\mu\left(R_{*}\right) \leqslant \mu_{0}$. Consequently, from (17) it follows that

$$
-\left|\left\langle A_{0}\left(u_{n+1}+t\left(u_{n}-u_{n+1}\right)\right)-A_{0} u_{n},\left(u_{n}-u_{n+1}\right)\right\rangle\right| \geqslant-\mu_{0} \Phi\left(\left\|u_{n}-u_{n+1}\right\|_{V}\right)\left\|u_{n}-u_{n+1}\right\|_{V}
$$

Furthermore, similarly to $[2,9]$ from $(7)$ we have

$$
\begin{aligned}
F\left(u_{n}\right)-F\left(u_{n+1}\right)= & \int_{0}^{1}\left\langle A_{0}\left(u_{n+1}+t\left(u_{n}-u_{n+1}\right)\right), u_{n}-u_{n+1}\right\rangle d t-\left\langle f, u_{n}-u_{n+1}\right\rangle+F_{1}\left(u_{n}\right)-F_{1}\left(u_{n+1}\right) \\
= & \int_{0}^{1}\left\langle A_{0}\left(u_{n+1}+t\left(u_{n}-u_{n+1}\right)\right)-A_{0} u_{n}, u_{n}-u_{n+1}\right\rangle d t-\left\langle f-A_{0} u_{n}, u_{n}-u_{n+1}\right\rangle \\
& +F_{1}\left(u_{n}\right)-F_{1}\left(u_{n+1}\right) .
\end{aligned}
$$


Hence, using (11) with $v=u_{n}$ and (9), (18), we obtain

$$
\begin{aligned}
F\left(u_{n}\right)-F\left(u_{n+1}\right) \geqslant & -\int_{0}^{1}\left|\left\langle A_{0}\left(u_{n+1}+t\left(u_{n}-u_{n+1}\right)\right)-A_{0} u_{n},\left(u_{n}-u_{n+1}\right)\right\rangle\right| d t \\
& +\tau^{-1}\left[\tau\left\langle f-A_{0} u_{n}, u_{n+1}-u_{n}\right\rangle+\tau\left(F_{\varepsilon_{n}}\left(u_{n}\right)-F_{\varepsilon_{n}}\left(u_{n+1}\right)\right)\right] \\
& +\left(F_{1}\left(u_{n}\right)-F_{\varepsilon_{n}}\left(u_{n}\right)\right)+\left(F_{\varepsilon_{n}}\left(u_{n+1}\right)-F_{1}\left(u_{n+1}\right)\right) \\
\geqslant & -\mu_{0} \Phi\left(\left\|u_{n}-u_{n+1}\right\|_{V}\right)\left\|u_{n}-u_{n+1}\right\|_{V} \\
& +\tau^{-1}\left\langle J\left(u_{n+1}-u_{n}\right), u_{n+1}-u_{n}\right\rangle-2 c\left(\varepsilon_{n}\right) \\
\geqslant & \lambda \Phi\left(\left\|u_{n}-u_{n+1}\right\|_{V}\right)\left\|u_{n}-u_{n+1}\right\|_{V}-2 c\left(\varepsilon_{n}\right), \quad \lambda=\frac{1}{\tau}-\mu_{0}>0 .
\end{aligned}
$$

Thus, we have

$$
F\left(u_{n+1}\right) \leqslant F\left(u_{n}\right)+2 c\left(\varepsilon_{n}\right) .
$$

Adding these inequalities at $n=0,1, \ldots, N-1$, we obtain

$$
F\left(u_{n+1}\right) \leqslant F\left(u_{0}\right)+2 \sum_{n=0}^{N} c\left(\varepsilon_{i}\right) \leqslant F\left(u_{0}\right)+2 \sigma,
$$

i.e., $u_{n+1} \in S_{0}$. Statement (15) has been proved.

We need the following result (see [21, p. 93]):

Lemma 2.1. Let the numerical sequence $\left\{a_{k}\right\}_{k=1}^{\infty}$ satisfy the conditions

$$
a_{k+1} \leqslant a_{k}+\delta_{k}, \delta_{k} \geqslant 0, k=1,2, \ldots, \quad \sum_{k=1}^{+\infty} \delta_{k}<+\infty .
$$

Then there exists

$$
\lim _{k \rightarrow+\infty} a_{k}<+\infty
$$

If, in addition, this sequence $\left\{a_{k}\right\}_{k=1}^{\infty}$ is lower-bounded, then $\lim _{k \rightarrow+\infty} a_{k}$ is finite.

The conditions of Lemma 2.1 for the sequence $\left\{F\left(u_{n}\right)\right\}$ are true because of (13), $(20)$. Indeed, from the definition of the functional $F$ given in (6) we have

$$
F(u) \geqslant-\mu\left(\|u\|_{V}\right) \Phi\left(\|u\|_{V}\right)\|u\|_{V}-\gamma^{*}\|u\|_{V}-\|f\|_{V^{*}}\|u\|_{V}+F_{1}(0)-\left\|A_{0}(0)\right\|_{V^{*}}\|u\|_{V} .
$$

From this inequality, the boundedness of the operator $A_{0}$ and the boundedness of the iterative sequence it follows that the sequence $\left\{F\left(u_{n}\right)\right\}_{n=1}^{\infty}$ is bounded. It means that the numerical sequence $\left\{F\left(u_{n}\right)\right\}_{n=1}^{\infty}$ has a finite limit. Therefore, from (19) we have

$$
\lim _{n \rightarrow+\infty} \lambda \Phi\left(\left\|u_{n}-u_{n+1}\right\|_{V}\right)\left\|u_{n}-u_{n+1}\right\|_{V}=0 .
$$

From this equality, by virtue of the continuity and the strict increase of the function $\Phi$, it follows that

$$
\lim _{n \rightarrow+\infty}\left\|u_{n}-u_{n+1}\right\|_{V}=0
$$


Furthermore, for an arbitrary function $v \in M$ from (11) we obtain

$$
\begin{aligned}
\left\langle A_{0} u_{n}, u_{n}-v\right\rangle \leqslant & \left\langle A_{0} u_{n}, u_{n}-u_{n+1}\right\rangle+\frac{1}{\tau}\left\langle J\left(u_{n+1}-u_{n}\right), v-u_{n+1}\right\rangle+\left(F_{\varepsilon_{n}}(v)-F_{\varepsilon_{n}}\left(u_{n}\right)\right) \\
& +\left(F_{\varepsilon_{n}}\left(u_{n}\right)-F_{\varepsilon_{n}}\left(u_{n+1}\right)\right)+\left\langle f, u_{n}-v\right\rangle+\left\langle f, u_{n+1}-u_{n}\right\rangle \\
\leqslant & \left(\left\|A_{0} u_{n}\right\|_{V^{*}}+\|f\|_{V^{*}}+\gamma^{*}\right)\left\|u_{n+1}-u_{n}\right\|_{V} \\
& +\frac{1}{\tau} \Phi\left(\left\|u_{n+1}-u_{n}\right\|_{V}\right)\left\|v-u_{n+1}\right\|_{V}+\left(F_{\varepsilon_{n}}(v)-F_{\varepsilon_{n}}\left(u_{n}\right)\right)+\left\langle f, u_{n}-v\right\rangle \\
\leqslant & C_{v}\left(\Phi\left(\left\|u_{n+1}-u_{n}\right\|_{V}\right)+\left\|u_{n+1}-u_{n}\right\|_{V}\right)+\left(F_{1}(v)-F_{1}\left(u_{n}\right)\right) \\
& +\left\langle f, u_{n}-v\right\rangle+2 c\left(\varepsilon_{n}\right),
\end{aligned}
$$

where $C_{v}$ is a positive constant that depends on $v$.

From the boundedness of the iterative sequence and reflexivity of the $V$ space there follows the existence of the subsequence $\left\{u_{n_{k}}\right\}_{k=1}^{\infty}$, which converges weakly to $u^{*}$ in $V$ as $k \rightarrow+\infty$. Let us see that for this subsequence inequality (3) is satisfied. From (22) with $v=u^{*}$, taking into account (21) and the weak lower semi-continuity of the functional $F_{1}$, we obtain

$$
\begin{aligned}
\limsup _{k \rightarrow \infty}\left\langle A_{0} u_{n_{k}}, u_{n_{k}}-u^{*}\right\rangle \leqslant \limsup _{k \rightarrow \infty} C_{u^{*}}\left(\Phi\left(\left\|u_{n_{k}+1}-u_{n_{k}}\right\|_{V}\right)+\left\|u_{n_{k}+1}-u_{n_{k}}\right\|_{V}\right) \\
+\limsup _{k \rightarrow \infty}\left(F_{1}\left(u^{*}\right)-F_{1}\left(u_{n_{k}}\right)\right)+\limsup _{k \rightarrow \infty}\left\langle f, u_{n_{k}}-u^{*}\right\rangle+2 \limsup _{k \rightarrow \infty} c\left(\varepsilon_{n_{k}}\right) \leqslant 0 .
\end{aligned}
$$

We show that $u^{*}$ is a solution of problem (8). From (22) for an arbitrary function $v \in M$ we have

$$
\begin{aligned}
C_{v}\left[\Phi\left(\left\|u_{n_{k}+1}-u_{n_{k}}\right\|_{V}\right)\right. & \left.+\left\|u_{n_{k}+1}-u_{n_{k}}\right\|_{V}\right] \\
& \geqslant\left\langle A_{0} u_{n_{k}}, u_{n_{k}}-v\right\rangle+\left(F_{1}\left(u_{n_{k}}\right)-F_{1}(v)\right)+\left\langle f, u_{n_{k}}-v\right\rangle-2 c\left(\varepsilon_{n_{k}}\right),
\end{aligned}
$$

consequently, by virtue of $(21)$, the weak lower semi-continuity of the functional $F_{1}$, and the pseudomonotonicity of the operator $A_{0}$ (inequality (4)) we have

$$
\begin{aligned}
0 & =\liminf _{k \rightarrow \infty} C_{v}\left[\Phi\left(\left\|u_{n_{k}+1}-u_{n_{k}}\right\|_{V}\right)+\left\|u_{n_{k}+1}-u_{n_{k}}\right\|_{V}\right]+2 c\left(\varepsilon_{n_{k}}\right) \\
& \geqslant \liminf _{k \rightarrow \infty}\left\langle A_{0} u_{n_{k}}, u_{n_{k}}-v\right\rangle+\liminf _{k \rightarrow \infty}\left(F_{1}\left(u_{n_{k}}\right)-F_{1}(v)\right)+\liminf _{k \rightarrow \infty}\left\langle f, v-u_{n_{k}}\right\rangle \\
& \geqslant\left\langle A_{0} u^{*}, u^{*}-v\right\rangle+F_{1}\left(u^{*}\right)-F_{1}(v)+\left\langle f, v-u^{*}\right\rangle,
\end{aligned}
$$

i.e., $u^{*}$ is the solution of the variational inequality (8).

Remark 2.1. If the subsequence $\left\{u_{n_{k}}\right\}_{k=0}^{\infty}$ of the iterative sequence $\left\{u_{n}\right\}_{n=0}^{\infty}$ converges weakly to $u^{*}$ in $V$ as $k \rightarrow+\infty$, then the following equality is true:

$$
\lim _{k \rightarrow+\infty}\left\langle A_{0} u_{n_{k}}-A_{0} u^{*}, u_{n_{k}}-u^{*}\right\rangle=0 .
$$

\section{The case of a Hilbert space}

We consider another case where the space $V$ is a Hilbert space. We denote by $(\cdot, \cdot)_{V}$ the scalar product in it and identify $V^{*}$ with $V$. Let, further, the operator $A_{0}$ instead of (2) satisfy the condition

$$
\left\|A_{0} u-A_{0} v\right\|_{V}^{2} \leqslant d_{0}\left(A_{0} u-A_{0} v, u-v\right)_{V}, \quad d_{0}>0 \quad \forall u, v \in V .
$$

We observe that inequality (2) is a sequence of inequality (23) with $\mu(\xi)=d_{0}, \Phi(\xi)=\xi$.

We shall prove the following. 
Theorem 3.1. Let $u_{0}$ be an arbitrary element of $M$. For $n=0,1,2, \ldots$ we define $u_{n+1} \in M$ as a solution of the variational inequality

$$
\left(u_{n+1}-u_{n}, v-u_{n+1}\right)_{V}+\tau\left(F_{\varepsilon_{n}}(v)-F_{\varepsilon_{n}}\left(u_{n+1}\right)\right) \geqslant \tau\left(f-A_{0} u_{n}, v-u_{n+1}\right)_{V} \quad \forall v \in M,
$$

where $0<\tau<\tau_{0}=2 / d_{0}$. Then the iterative sequence $\left\{u_{n}\right\}_{n=0}^{\infty}$ converges weakly in $V$ for the solution of problem (8).

Proof. Following the proof of Theorem 2.1 with explicit changes, it is easy to check that the sequence $\left\{u_{n}\right\}_{n=0}^{\infty}$ is bounded and any of its weak limit points is the solution of problem (8).

Let $u^{*}$ be an arbitrary solution of problem (8), i.e.,

$$
\tau\left(F_{1}(v)-F_{1}\left(u^{*}\right)\right) \geqslant \tau\left(f-A_{0} u^{*}, v-u^{*}\right)_{V} \quad \forall v \in M .
$$

We substitute into the last inequality $v=u_{n+1}, v=u^{*}$ into (24) and add the obtained inequalities. We have

$$
\begin{aligned}
\left(w_{n+1}, w_{n+1}\right)_{V} \leqslant & \left(w_{n}, w_{n+1}\right)_{V}-\tau\left(A_{0} u_{n}-A_{0} u^{*}, w_{n+1}\right)_{V} \\
& +\tau\left(F_{\varepsilon_{n}}\left(u^{*}\right)-F_{\varepsilon_{n}}\left(u_{n+1}\right)\right)+\tau\left(F_{1}\left(u_{n+1}\right)-F_{1}\left(u^{*}\right)\right),
\end{aligned}
$$

where $w_{n}=u_{n}-u^{*}, n=0,1, \ldots$ Hence, using (10), we obtain

$$
\left\|w_{n+1}\right\|_{V} \leqslant\left\|w_{n}-\tau\left(A_{0} u_{n}-A_{0} u^{*}\right)\right\|_{V}+2 \tau c\left(\varepsilon_{n}\right)
$$

Then

and finally

$$
\left\|w_{n+1}\right\|_{V}^{2} \leqslant \frac{1}{2}\left\|w_{n}-\tau\left(A_{0} u_{n}-A_{0} u^{*}\right)\right\|_{V}^{2}+\frac{1}{2}\left\|w_{n+1}\right\|_{V}^{2}+2 \tau c\left(\varepsilon_{n}\right)
$$

$$
\left\|w_{n+1}\right\|_{V}^{2} \leqslant\left\|w_{n}-\tau\left(A u_{n}-A u^{*}\right)\right\|_{V}^{2}+4 \tau c\left(\varepsilon_{n}\right) .
$$

From inequality (25) we obtain

$$
\left\|w_{n+1}\right\|_{V}^{2} \leqslant\left\|w_{n}\right\|_{V}^{2}-2 \tau\left(A_{0} u_{n}-A_{0} u^{*}, u_{n}-u^{*}\right)_{V}+\tau^{2}\left\|A_{0} u_{n}-A_{0} u^{*}\right\|_{V}^{2}+4 \tau c\left(\varepsilon_{n}\right) .
$$

Using inequality (23), from (26) we have

$$
\left\|w_{n+1}\right\|_{V}^{2} \leqslant\left\|w_{n}\right\|_{V}^{2}-\tau\left(2-d_{0} \tau\right)\left(A_{0} u_{n}-A_{0} u^{*}, u_{n}-u^{*}\right)_{V}+4 \tau c\left(\varepsilon_{n}\right) .
$$

Thus, when $0<\tau \leqslant 2 / d_{0}$ the numerical sequence $\left\{\left\|u_{n}-u^{*}\right\|_{V}\right\}_{n=1}^{\infty}$ satisfies the conditions of Lemma 2.1 and, consequently, there is a finite limit of this numerical sequence as $n \rightarrow+\infty$

$$
\lim _{n \rightarrow+\infty}\left\|u_{n}-u^{*}\right\|_{V}=\lambda_{u^{*}}
$$

We shall prove that all weak limit points of the sequence $\left\{u_{n}\right\}_{n=0}^{\infty}$ coincide. From this there will follow a weak convergence of the whole iterative sequence to a solution of problem (8).

Let $v, w$ be two weak limit points of the sequence $\left\{u_{n}\right\}_{n=0}^{\infty}$, i.e., there exist subsequences $\left\{u_{n_{k}}\right\}_{k=0}^{\infty},\left\{u_{m_{k}}\right\}_{k=0}^{\infty}$ that converge weakly in $V$ to $v$ and $w$ respectively as $k \rightarrow+\infty$. By virtue of Theorem 2.1 the elements $v$ and $w$ are solutions of problem (8), consequently, for the whole sequence $\left\{u_{n}\right\}_{n=0}^{\infty}$ relations (27) with $u^{*}=v$ and $u^{*}=w$ hold, i.e.,

$$
\lim _{n \rightarrow+\infty}\left\|u_{n}-v\right\|_{V}=\lambda_{v}, \quad \lim _{n \rightarrow+\infty}\left\|u_{n}-w\right\|_{V}=\lambda_{w} .
$$


Obviously, these relations also hold for any subsequences of the sequence $\left\{u_{n}\right\}_{n=0}^{\infty}$, in particular for the subsequences $\left\{u_{n_{k}}\right\}_{k=0}^{\infty},\left\{u_{m_{k}}\right\}_{k=0}^{\infty}$

$$
\lim _{k \rightarrow+\infty}\left\|u_{n_{k}}-v\right\|_{V}=\lambda_{v}, \quad \lim _{k \rightarrow+\infty}\left\|u_{m_{k}}-w\right\|_{V}=\lambda_{w}
$$

According $[17,18]$, we consider the numerical sequence $\left\{e_{k}\right\}_{k=0}^{\infty}$ defined by the formula

$$
e_{k}=\left\|u_{n_{k}}-v\right\|_{V}^{2}-\left\|u_{n_{k}}-w\right\|_{V}^{2}-\left\|u_{m_{k}}-v\right\|_{V}^{2}+\left\|u_{m_{k}}-w\right\|_{V}^{2}, \quad k=0,1,2, \ldots
$$

From (28) we have

$$
\lim _{k \rightarrow+\infty} e_{k}=0 .
$$

On the other hand, by the direct calculations it is easy to verify that

$$
e_{k}=-2\left(u_{n_{k}}-u_{m_{k}}, v-w\right)_{V}
$$

hence, by the weak convergence of the subsequences $\left\{u_{n_{k}}\right\}_{k=0}^{\infty},\left\{u_{m_{k}}\right\}_{k=0}^{\infty}$ in V to $v$ and $w$, respectively, as $k \rightarrow+\infty$ we obtain that

$$
\lim _{k \rightarrow+\infty} e_{k}=-2(v-w, v-w)_{V}=-2\|v-w\|_{V}^{2}
$$

Thus, we have $\|v-w\|_{V}=0$, i.e., $v=w$.

\section{Application to seepage problems with a limiting gradient in a domain with a semipermeable boundary}

Let $\Omega$ be a bounded domain in $R^{m}, m \geqslant 1$, with a Lipshitz continuous boundary $\Gamma, \xi \rightarrow$ $g\left(\xi^{2}\right) \xi$ is a function defining the filtration law. We assume that $g\left(\xi^{2}\right) \xi=g_{0}\left(\xi^{2}\right) \xi+g_{1}\left(\xi^{2}\right) \xi$ and the following conditions are satisfied:

$$
\begin{array}{ll}
g_{i}\left(\xi^{2}\right) \xi \geqslant 0 \text { for } \xi \geqslant 0, & g_{i}\left(\xi^{2}\right) \xi=0 \text { for } \xi \leqslant \beta, \quad i=0,1, \\
g_{0}\left(\xi^{2}\right) \xi \text { is continuous, } & \left(g_{0}\left(\xi^{2}\right) \xi\right)_{\xi}^{\prime}>0 \text { for } \xi \geqslant \beta,
\end{array}
$$

$\beta \geqslant 0$ is a limiting gradient,

there exist $c_{0}, c_{1}, c_{2}>0, p>1$ such that

$$
\begin{gathered}
c_{1}(\xi-\beta)^{p-1} \leqslant g_{0}\left(\xi^{2}\right) \xi \leqslant c_{2}(\xi-\beta)^{p-1} \quad \text { for } \quad \xi \geqslant \beta, \\
\left(g_{0}\left(\xi^{2}\right) \xi-g_{0}\left(\eta^{2}\right) \eta\right) /(\xi-\eta) \leqslant c_{0}(1+\xi+\eta)^{p-2} \quad \forall \xi, \eta \in R^{1}>0, \\
g_{1}\left(\xi^{2}\right) \xi=\vartheta \quad \text { for } \xi>\beta .
\end{gathered}
$$

Furthermore let $\Gamma=\Gamma_{0} \cup \Gamma_{1}$, mes $\Gamma_{0}>0$, mes $\Gamma_{1}>0, V=\left\{v \in W_{p}^{(1)}(\Omega): v(x)=\right.$ $\left.0, x \in \Gamma_{0}\right\}, M=\left\{v \in V: v(x) \geqslant 0, x \in \Gamma_{1}\right\}$ ( $\Gamma_{1}$ is a semipermeable part of the boundary), $A_{0}: V \rightarrow V^{*}$ is an operator generated by the form

$$
\left\langle A_{0} u, v\right\rangle=\int_{\Omega} g_{0}\left(|\nabla u|^{2}\right)(\nabla u, \nabla v) d x, \quad u, v \in V,
$$

where $(\cdot, \cdot)$ and $|\cdot|$ are the inner production and norm in $R^{m}$ respectively. 
We also define the functional $F_{1}: V \rightarrow R^{1}$ by the relation

$$
F_{1}(v)=\int_{\Omega} \int_{0}^{|\nabla v|} g_{1}\left(\xi^{2}\right) \xi d \xi d x=\vartheta \int_{\Omega}(|\nabla v|-\beta)^{+} d x, \quad a^{+}=(|a|+a) / 2 .
$$

We call the function $u \in M$ the solution of the seepage problem of an incompressible liquid which is the solution of the variational inequality (8) (see $[3,12]$ ).

We investigate some solution properties of problem (8). We need the following lemma (see [5]).

Lemma 4.1. Let the function $\psi: R^{1} \rightarrow R^{1}$ be such that $\psi\left(x^{2}\right) x$ monotonely increases when $x \geqslant 0$. Then the function $H: R^{m} \times R^{m} \rightarrow R^{1}$,

$$
H(\xi, \zeta) \equiv\left(\psi\left(|\xi|^{2}\right) \xi-\psi\left(|\zeta|^{2}\right) \zeta, \xi-\zeta\right)
$$

is non-negative. Moreover, if $\psi\left(x^{2}\right)=0$ when $0 \leqslant x \leqslant \alpha, \alpha>0, \psi\left(x^{2}\right) x$ is strictly increasing when $x>\alpha$ then for the case where $H(\xi, \zeta)=0$ :

$$
\text { 1) } \xi=\zeta \text { for }|\xi|>\alpha, \quad \text { 2) }|\zeta| \leqslant \alpha \text { for }|\xi| \leqslant \alpha \text {. }
$$

Lemma 4.2. Let for $\eta_{1}, \eta_{2} \in V$ the equality

$$
\left\langle A_{0} \eta_{1}-A_{0} \eta_{2}, \eta_{1}-\eta_{2}\right\rangle=0
$$

hold. Then with an accuracy to the set of measure zero the following equalities are satisfied:

$$
\Omega_{\eta_{1}}^{+}=\Omega_{\eta_{2}}^{+}=\Omega^{*}, \quad \Omega_{\eta}^{+}=\{x \in \Omega:|\nabla \eta(x)|>\beta\},
$$

besides,

$$
\begin{aligned}
\nabla \eta_{1} & =\nabla \eta_{2} \quad \text { almost everywhere on } \Omega^{*}, \\
g\left(\left|\nabla \eta_{1}\right|^{2}\right) \nabla \eta_{1} & =g\left(\left|\nabla \eta_{2}\right|^{2}\right) \nabla \eta_{2} \quad \text { almost everywhere on } \Omega .
\end{aligned}
$$

Proof. By virtue of Lemma 4.1 with $\psi=g$ we have

$$
0=\left\langle A_{0} \eta_{1}-A_{0} \eta_{2}, \eta_{1}-\eta_{2}\right\rangle=\int_{\Omega} H\left(\nabla \eta_{1}, \nabla \eta_{2}\right) d x \geqslant 0
$$

i.e.,

$$
\int_{\Omega} H\left(\nabla \eta_{1}, \nabla \eta_{2}\right) d x=0
$$

The subintegral function is non-negative, hence $H\left(\nabla \eta_{1}, \nabla \eta_{2}\right)=0$ almost everywhere on $\Omega$. But then from statement 2) of Lemma 4.1 it follows that

$$
\Omega \backslash \Omega_{\eta_{1}}^{+}=\Omega \backslash \Omega_{\eta_{2}}^{+},
$$

from where (35) follows. From statement 1) of Lemma 4.1 we obtain that equality (36) holds.

From (35), (36) follow the equalities

$$
g\left(\left|\nabla \eta_{1}\right|^{2}\right) \nabla \eta_{1}=g\left(\left|\nabla \eta_{2}\right|^{2}\right) \nabla \eta_{2}
$$


almost everywhere on $\Omega^{*}$.

On the set $\Omega \backslash \Omega^{*}$ we have

$$
g\left(\left|\nabla \eta_{1}\right|^{2}\right) \nabla \eta_{1}=g\left(\left|\nabla \eta_{2}\right|^{2}\right) \nabla \eta_{2}=0
$$

i.e., equality (37) holds.

Theorem 4.1. The seepage velocity $v$, constructed by the solution u of problem (8) in accordance with the formula

$$
v=-g\left(|\nabla u|^{2}\right) \nabla u
$$

is defined uniquely; for any solutions $u_{1}, u_{2}$ of problem (8) with an accuracy to the set of measure zero the equality

$$
\Omega_{u_{1}}^{+}=\Omega_{u_{2}}^{+}=\Omega^{*}
$$

holds.

Proof. Let $u_{1}, u_{2}$ be two solutions of problem (8)

$$
\begin{array}{ll}
\left\langle A_{0} u_{1}, \eta-u_{1}\right\rangle+F_{1}(\eta)-F_{1}\left(u_{1}\right) \geqslant\left\langle f, \eta-u_{1}\right\rangle & \forall \eta \in M, \\
\left\langle A_{0} u_{2}, \eta-u_{2}\right\rangle+F_{1}(\eta)-F_{1}\left(u_{2}\right) \geqslant\left\langle f, \eta-u_{2}\right\rangle & \forall \eta \in M .
\end{array}
$$

Putting $\eta=u_{2}$ in (38), $\eta=u_{1}$ in (39) and adding the obtained inequalities, taking into account the monotonocity of the operator $A_{0}$ that follows from Lemma 4.1, we have

$$
\left\langle A_{0} u_{1}-A_{0} u_{2}, u_{1}-u_{2}\right\rangle=0 .
$$

The statement of the theorem follows now from Lemma 4.2.

By virtue of conditions (29) - (33) the operator $A_{0}$ is monotone, coercive, potential (thus, equality (1) holds) (see $[3,16]$ ) and satisfies condition (2) (see [2]) with the functions $\Phi, \mu$, that are defined by the formulas

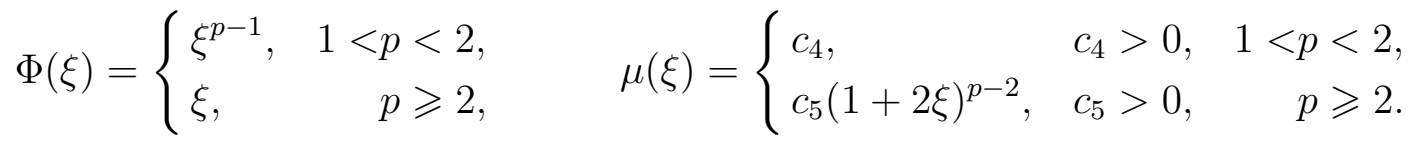

From the definition of the functional $F_{1}$, the second of the conditions (29), and equality (33) the next lemma follows (see [4]).

Lemma 4.3. The functional $F_{1}$ must satisfy the condition

$$
\left|F_{1}(v)-F_{1}(u)\right| \leqslant \vartheta\|\nabla(v-u)\|_{L_{1}} .
$$

From Lemma 4.3 it follows that the functional $F_{1}$ is Lipshitz continuous.

We now define the regularized function

$$
g_{1 \varepsilon}\left(\xi^{2}\right) \xi=\left\{\begin{array}{lr}
0, & \xi \leqslant \beta-\varepsilon \\
\vartheta(\xi-\beta+\varepsilon) / \varepsilon, & \beta-\varepsilon \leqslant \xi \leqslant \beta \\
\vartheta, & \xi \geqslant \beta .
\end{array}\right.
$$

This function generates the corresponding regularized functional $F_{1 \varepsilon}$. We shall prove the following. 
Lemma 4.4. Let the function $g_{1 \varepsilon}\left(\xi^{2}\right) \xi$ be defined by (40). Then the functional $F_{1 \varepsilon}$ satisfies condition (9) with $c(\varepsilon)=2 \varepsilon$ mes $\Omega$.

Proof. We denote $\Omega_{1 \varepsilon \eta}=\{x \in \Omega: \beta-\varepsilon \leqslant|\nabla \eta| \leqslant \beta\}, \quad \Omega_{1 \eta}=\{x \in \Omega:|\nabla \eta|>\beta\}$. On $\Omega_{1 \varepsilon \eta}$ we have $0 \leqslant \beta-|\nabla \eta| \leqslant-\varepsilon$, hence,

$$
\begin{aligned}
\left|F_{1 \varepsilon}(\eta)-F_{1}(\eta)\right| \leqslant & \left|\int_{\Omega_{1 \varepsilon \eta}} \int_{\beta-\varepsilon}^{|\nabla \eta|} g_{1 \varepsilon}\left(\xi^{2}\right) \xi d \xi d x\right| \\
& +\left|\int_{\Omega_{1 \eta}}\left[\int_{\beta-\varepsilon}^{\beta} g_{1 \varepsilon}\left(\xi^{2}\right) \xi d \xi+\int_{\beta}^{|\nabla \eta|}\left(g_{1 \varepsilon}\left(\xi^{2}\right) \xi-g_{1}\left(\xi^{2}\right) \xi\right) d \xi\right] d x\right| \\
= & \left|\frac{\vartheta}{2 \varepsilon} \int_{\Omega_{1 \varepsilon \eta}}(|\nabla \eta|-\beta+\varepsilon)^{2} d x\right|+\frac{\vartheta \varepsilon}{2} \operatorname{mes} \Omega_{1 \eta} \\
\leqslant & \frac{\vartheta}{2 \varepsilon} \int_{\Omega_{1 \varepsilon \eta}}\left[(|\nabla \eta|-\beta)^{2}+2 \varepsilon(|\nabla \eta|-\beta)\right] d x+\frac{\vartheta \varepsilon}{2} \int_{\Omega_{1 \varepsilon \eta}} d x \\
& +\frac{\vartheta}{2 \varepsilon} 3 \varepsilon^{2} \operatorname{mes} \Omega_{1 \eta \varepsilon}+\frac{\vartheta \varepsilon}{2} \operatorname{mes} \Omega \leqslant 2 \varepsilon \operatorname{mes} \Omega .
\end{aligned}
$$

When $p=2$ the space $\mathrm{V}$ is a Hilbert space, in [16] inequality (23) is determined for the operator $A_{0}$ defined in (34).

Thus, for the seepage problems under consideration the conditions of the previous sections of this paper are satisfied and, consequently, Theorems 2.1 and 3.1 hold.

Additional properties of the iterative sequence can be obtained with the help of the property of the kind of $(S)_{+}$(the property $(S)_{+}$is defined in [20]). Namely, in [5] it has been proved that if the sequence $v_{n}$ converges weakly to $v$ in $V$ and

$$
\lim _{n \rightarrow+\infty}\left\langle A_{0} v_{n}, v_{n}-v\right\rangle=0,
$$

then the sequence $\nabla v_{n}$ converges to $\nabla v$ in $\left[L_{p}\left(\Omega_{v}^{+}\right)\right]^{m}$, the sequence $G_{0}\left(\nabla v_{n}\right)$ converges to $G_{0}(\nabla v)$ in $\left[L_{q}(\Omega)\right]^{m}$ as $n \rightarrow+\infty$, where

$$
G_{0}(a)=g_{0}\left(|a|^{2}\right) a /|a|, \quad a \in R^{m} .
$$

From Theorem 2.1, according to [5], it is easy to see that the following theorem holds.

Theorem 4.2. Let $u$ be any solution of the seepage problem (8) and $\left\{u_{n}\right\}_{n=1}^{\infty}$ is the iterative sequence constructed according to the conditions of Theorem 2.1. Then the sequence $\nabla u_{n}$ converges to $\nabla u$ in $\left[L_{p}(\Omega(u))\right]^{m}$ and the sequence $G_{0}\left(\nabla u_{i}\right)$ converges to $G_{0}(\nabla u)$ in $\left[L_{q}(\Omega)\right]^{m}$ as $n \rightarrow+\infty, q=p /(p-1)$.

\section{Acknowledgment}

We wish to thank the anonymous referee for helpful remarks. 


\section{References}

[1] I. B. Badriev and M. M. Karchevskiy, Application of the duality method for the solution of nonlinear seepage problems with limit gradient, Differ. Uravn., 18(1982), No. 7, pp. 1133-1144, in Russian.

[2] I. B. Badriev and M. M. Karchevskiy, On the convergence of the iterative process in the Banach space, in: Research in Applied Mathematics, Kazan University Press, Kazan, issue 17, 1990, pp. 3-15, in Russian.

[3] I. B. Badriev and R. R. Shagidullin, Investigation of one-dimensional equations of the static state of a soft shell and of an algorithm for their solution, Izv. Vyssh. Uchebn. Zaved., Mat., 1992, No. 1, pp. 7-17, in Russian.

[4] I. B. Badriev and O. A. Zadvornov, On the convergence of the iterative process for the solution of a variational inequality of the second order, in: Research in Applied Mathematics, Kazan Mathematical Society Press, Kazan, Issue 22, 1990, pp.3-15, in Russian.

[5] I. B. Badriev and O. A. Zadvornov, Investigation of the convergence of the iterative process for equations with degenerative operators, Differ. Uravn., 32(1996), No. 7, pp. 898-901, in Russian.

[6] I. B. Badriev, O. A. Zadvornov, and A. M. Saddeek, The research of convergence of iterative methods for solving of some variational inequalities of the second kind, Differ. Uravn., 37(2001), No. 7, pp. 1009-1016, in Russian.

[7] I. Ekeland and R. Temam, Convex Analysis and Variational Problems, Mir, Moscow, 1979, in Russian.

[8] D. Gabay and B. Merscier, A dual algorithm for the solution of nonlinear variational problems via finite element approximations, Computer Math. Appl., 2(1976), pp. 17-40.

[9] Kh. Gayevskiy, K. Greger, and K. Zacharias, Nonlinear Operator Equations and Operator Differential Equations, Mir, Moscow, 1978, in Russian.

[10] R. Glowinski, J. L. Lions, and R. Trémolières, Analyse Numérique des Inéquations Variationnelles, Dunod, Paris, 1976.

[11] V. D. Glushenkov, On an equation of the nonlinear seepage theory, in: Applied Mathematics in Scientific and Technical Problems, Kazan University Press, Kazan, 1976, pp. 3-15, in Russian.

[12] A. V. Lapin, On the research of some problems of the nonlinear seepage theory, Zh. Vychisl. Mat. Fiz., 19(1979), No. 3, pp. 689-700, in Russian.

[13] J. L. Lions, Some Methods for Solving Nonlinear Boundary Problems, Mir, Moscow, 1972, in Russian.

[14] P. L. Lions and B. Merscier, Splitting algorithms for the sum of two nonlinear operators, SIAM J. Numer. Anal., 16(1979), No. 6, pp. 964-979.

[15] A. D. Lyashko, I. B. Badriev, and M. M. Karchevskiy, On the variational method for equations with discontinuous monotone operators, Izv. Vyssh. Uchebn. Zaved., Mat., 1978, No. 11, pp.63-69, in Russian.

[16] A. D. Lyashko and M. M. Karchevskiy, On the solution of some nonlinear problems of the seepage theory , Izv. Vyssh. Uchebn. Zaved., Mat., 1975, No. 6, pp. 73-81, in Russian.

[17] S. Maruster, The solution by iteration of nonlinear equations in Hilbert spaces, Proc. Amer. Math. Soc., 63(1977), No. 1, pp. 69-73.

[18] Z. Opial, Weak convergence of the sequence of successive approximations for nonexpansive mappings, Bull. Amer. Math. Soc., 73(1967), pp. 591-597.

[19] Résolution Numériques de Problèmes aux Limites par des Mméthodes de Lagrangien Augmenté (M. Fortin, R. Glowinski, eds.), Dunod, Paris, 1983, 320 p.

[20] I. V. Skripnik, Methods of Investigating Nonlinear Elliptic Boundary Problems, Nauka, Moscow, 1990, in Russian.

[21] F. P. Vasil'ev, Numerical Methods for Solving Extremal Problemes, Nauka, Moscow, 1988, in Russian. 